\title{
1 Live imaging of Yersinia translocon formation and immune recognition in host cells
}

2 Maren Rudolph ${ }^{1}$, Alexander Carsten ${ }^{1}$, Martin Aepfelbacher ${ }^{1 *}$ and Manuel Wolters ${ }^{1 *}$

$3 *$ equal contribution

4 Institute of Medical Microbiology, Virology and Hygiene, University Medical Center Hamburg Eppendorf,

5 Martinistraße 52, 20251 Hamburg, Germany

$6 \quad$ Correspondence: m.aepfelbacher@uke.de 


\section{Abstract}

8 Yersinia enterocolitica employs a type three secretion system (T3SS) to translocate immunosuppressive

9 effector proteins into host cells. To this end, the T3SS assembles a translocon/pore complex composed of

10 the translocator proteins YopB and YopD in host cell membranes serving as an entry port for the

11 effectors. The translocon is formed in a Yersinia-containing pre-phagosomal compartment that is

12 connected to the extracellular space. As the phagosome matures, the translocon and the membrane

13 damage it causes are recognized by the cell-autonomous immune system. We infected cells in the

14 presence of fluorophore-labeled ALFA-tag-binding nanobodies with a $Y$. enterocolitica strain expressing

15 YopD labeled with an ALFA-tag. Thereby we could record the integration of YopD into translocons and its

16 intracellular fate in living host cells. YopD was integrated into translocons around 2 min after uptake of

17 the bacteria into a phosphatidylinositol-4,5-bisphosphate enriched pre-phagosomal compartment and

18 remained there for $27 \mathrm{~min}$ on average. Damaging of the phagosomal membrane as visualized with

19 recruitment of GFP-tagged galectin-3 occurred in the mean around 14 min after translocon formation.

20 Shortly after recruitment of galectin-3, guanylate-binding protein 1 (GBP-1) was recruited to

21 phagosomes, which was accompanied by a decrease in the signal intensity of translocons, suggesting

22 their degradation. In sum, we were able for the first time to film the spatiotemporal dynamics of Yersinia

23 T3SS translocon formation and degradation and its sensing by components of the cell-autonomous

24 immune system. 


\section{Introduction}

Type three secretion systems (T3SSs) are multi-component, syringe-like nanomachines that enable the

27 translocation of bacterial effector proteins across the bacterial envelope into eukaryotic host cells.

28 Numerous human pathogenic bacteria such as Yersinia, Pseudomonas, Chlamydia, Shigella and

Salmonella employ T3SS-mediated effector translocation to manipulate a variety of cellular processes,

ultimately determining the nature of interaction with their hosts. T3SS effector proteins are diverse in

structure and biochemical activities and vary considerably between species. In contrast, the T3SS

machinery - also known as the injectisome - is highly conserved across different bacterial species and has

been the subject of intensive structural and functional investigation [1-3].

Injectisomes can be separated into defined substructures such as the sorting platform, the export

apparatus, the needle complex, the tip complex and the translocon (Fig 1A). The needle complex is a

multi-ring cylindrical structure embedded in the bacterial envelope connected with a 30-70 nm long

needle filament, forming a narrow channel, through which the translocator and effector proteins pass in

an unfolded state $[4,5]$. The needle at its distal end transitions into the tip complex, which consists of

several copies of a hydrophilic translocator protein (5 copies of LcrV in Yersinia) [6]. The tip complex is

involved in host cell sensing and regulates the assembly of the translocon/pore complex [7].

41 The translocon of all investigated T3SSs consists of two hydrophobic translocator proteins, a major (YopB

in Yersinia) and a minor translocator (YopD in Yersinia) harboring one and two transmembrane domains,

respectively $[8,9]$. The two translocators are thought to form a heteromultimeric ring structure with an

44 inner opening of approximately $2-4 \mathrm{~nm}$ in the host cell membrane [10-12]. Despite the central role of the

47 translocon can only be studied when inserted into host cell membranes, up to now hindered its 
49 In a recent cryo-electron tomography study the host cell membrane embedded translocon of Salmonella

50 enterica minicells was found to have a total diameter of $13.5 \mathrm{~nm}$ [13]. In our previous work translocons

51 of Yersinia enterocolitica were imaged by super resolution immunofluorescence techniques (STED, SIM)

52 using antibodies against the translocator proteins YopB and YopD. Thereby, the host cellular context that

53 promotes translocon formation could be investigated, revealing that the translocons are formed upon

54 uptake of the bacteria into a phosphatidylinositol-4,5-bisphosphate (PIP2) - enriched pre-phagosomal

55 compartment/prevacuole, which is still connected to the extracellular space [14].

While these approaches provided a considerable degree of spatial resolution, none of them was suitable

57 for time resolved imaging of translocons. Live imaging of bacterium-host cell interactions using

58 fluorescence microscopy has become a key technology for understanding bacterial infection biology [15].

59 However, live imaging of translocon formation and processing in host cells has not yet been

60 accomplished. This is likely due to the elaborate und highly coordinated export of the hydrophobic

61 translocator proteins through the T3SS needle and their interaction with the tip complex before they

62 assemble a heteromultimeric translocon in the host cell membrane $[8,16]$. Thus, finding a label for

63 translocon proteins that is e.g., suited for live cell imaging and super resolution and at the same time

64 does not disturb translocon assembly has proven to be difficult. Fusion proteins of T3SS substrates with

65 fluorescent proteins like GFP were shown to be resistant to T3SS-mediated unfolding and block the

66 secretion path [17]. Several other tags (e.g. self-labeling enzymes Halo, CLIP, SNAP, split-GFP, 4Cys-tag/

67 FIAsH, iLOV) are secreted more effectively and have been used with varying degree of success for live

68 imaging of translocated effectors [18-22].

69 We here report the first live cell imaging data of Yersinia translocon formation, immune sensing and

70 processing by employing a Yersinia strain carrying a novel 13 amino acid peptide tag called ALFA-tag in

71 the minor hydrophobic translocator YopD [23]. These data provide novel insights on the spatiotemporal

72 dynamics and immune recognition of bacterial T3SS translocons. 


\section{Characterization of $Y$. enterocolitica strain WA-314 YopD-ALFA}

75 During infection with pathogenic yersiniae, a translocon/heteromultimeric pore complex composed of

76 the translocator proteins YopB and YopD is integrated into host cell membranes, serving as an entry gate

77 for the effector proteins (Fig 1A). In search of a method to visualize translocons in living cells using

78 fluorescence microscopy, we constructed strain WA-314 YopD-ALFA harboring a YopD variant in which

79 an ALFA-tag (plus linkers), was inserted between amino acids 194 and 195 (see Methods). The ALFA-tag

80 insertion site is supposedly located in the extracellular part of YopD after it has integrated into the host

81 cell membrane (Fig. 1B) [16]. The 13 amino acid long ALFA-tag can be bound with high affinity by specific

82 nanobodies (NbALFA) [23].

83 We first investigated whether WA-314 YopD-ALFA retains wild type functionalities by comparing its secretion-, translocon forming- and translocation capabilities as well as cytotoxic effect with the parental strain WA-314 (Fig. 1C - F). SDS-PAGE and Western blot showed similar levels of total secreted proteins and secreted YopD-ALFA in WA-314 YopD-ALFA when compared to WA-314 (YopD-ALFA levels were compared to YopD levels), suggesting that protein secretion is unaffected in WA-314 YopD-ALFA (Fig. 1C). Staining of WA-314 YopD-ALFA infected HeLa cells with fluorophore-labeled NbALFA revealed distinct fluorescence patches that were also detected by anti-YopD and anti-YopB antibodies (Fig. 1D). Such patches have recently been shown to represent clusters of translocons [14]. Further, WA-314 YopDALFA and WA-314 translocated similar amounts of a YopE $\beta$-lactamase fusion protein into host cells, as determined with a $\beta$-lactamase reporter system (Fig. 1E) [24-26]. In addition, rounding of HeLa cells was induced to a similar extent by infection with WA314 and WA-314 YopD-ALFA but not with WA314 $\triangle$ YopD. indicates that effector translocation into host cells is unaffected in WA-314 YopD-ALFA (Fig. 1F). We 


\section{Fluorescence staining of YopD-ALFA in pre-phagosomes, phagosomes and Yersinia cells}

99 In previous work we showed that translocon formation by $Y$. enterocolitica occurs in a specific pre-

100 phagosomal host cell compartment, previously referred to as prevacuole [14, 27]. The Yersinia-

101 containing pre-phagosome is characterized by a PIP2-enriched membrane and a narrow connection to

102 the extracellular space, which cannot be passed by large extracellular molecules such as antibodies (MW

103 approx. $150 \mathrm{kDa}$ ), but by small molecules like streptavidin (MW $53 \mathrm{kDa}$ ) [14, 27]. We therefore assumed

104 that it should be feasible to stain YopD-ALFA in newly formed translocons by adding fluorophore-labeled

105 NbALFA (MW $15 \mathrm{kDa}$ ) to fixed but unpermeabilized WA-314 YopD-ALFA infected cells. To test this notion

106 and further investigate the localization of YopD-ALFA in the course of cell infection, we sequentially

107 stained WA-314 YopD-ALFA infected HeLa cells without permeabilization (NbALFA-635), after

108 permeabilization of the HeLa cell membranes with digitonin (NbALFA-580) and after additional

109 permeabilization of the bacterial membranes with $0.1 \%$ Triton X-100 (NbALFA-488) (Fig 2A). In

110 unpermeabilized HeLa cells, patchy fluorescence signals associated with bacteria could be detected (Fig.

$1112 \mathrm{~A}$, left), confirming that translocon-associated YopD-ALFA can be accessed by extracellularly added

112 NbALFA. In digitonin-permeabilized HeLa cells, additional translocon signals could be found that were

113 not seen in unpermeabilized cells, indicating that these translocons resided in closed phagosomes (Fig.

$1142 \mathrm{~A}$, middle). After additional permeabilization of the bacterial membranes with Triton $\mathrm{X}-100$, the

115 intrabacterial pool of YopD-ALFA could be visualized in all bacteria, independent of whether they

116 displayed translocons (Fig. 2A, right). The diffuse distribution of intrabacterial YopD-ALFA is in clear

117 contrast to the patchy pattern of translocon-associated YopD-ALFA. To better resolve translocon

118 associated from intrabacterial YopD-ALFA, we employed super resolution STED microscopy (Fig. 2B).

119 While the intrabacterial distribution of YopD-ALFA remained diffuse also at this level of resolution, the

120 extrabacterial YopD-ALFA produced distinct signals with a lateral extent of about $40 \mathrm{~nm}$, which

121 previously were identified as single translocons [14]. Overall, differential YopD-ALFA staining allows to 
visualize Yersinia translocons located in pre-phagosomes and phagosomes, as well as the intrabacterial

123 YopD pool.

\section{Live imaging of Yersinia translocon formation during cell infection}

126 Given the ability to stain translocons in fixed and unpermeabilized cells by external addition of

127 fluorophore-labeled NbALFA, we hypothesized that this may also enable the recording of translocon

128 formation in living cells. To test this possibility, live HeLa cells expressing GFP-LifeAct and myc-Rac1Q61L

129 were infected with WA-314 YopD-ALFA in the presence of NbALFA-580 and imaged using spinning disc

130 microscopy with one acquisition per minute. myc-Rac1Q61L was overexpressed in the HeLa cells because

131 it strongly increases the percentage of bacteria forming translocons [14]. GFP-LifeAct was expressed to

132 visualize host cells and to enable the localization of the cell adhering bacteria. With this approach, 133 appearance and disappearance of fluorescence signals corresponding to translocon-associated YopD-

134 ALFA could be recorded over time (Fig. 3A and movie S1). From their first visible appearance (at 5 min in 135 Fig. 3A), the number and intensity of YopD-ALFA fluorescence signals peaked after about 20 min and 136 decreased thereafter. The mean overall lifespan of YopD-ALFA fluorescence signals, defined as their first 137 visible appearance until their complete vanishing, was determined to be $26.6+/-13$ min (mean +/- S.D.,

138 Fig. 3B). The disappearance of the fluorescence signals was most certainly not due to photo bleaching 139 because no decay of fluorescence was observed in recordings with considerably higher imaging 140 frequency (e.g acquisition rate: 3 per min in Fig. $3 C$ vs. 1 per min in Fig. 3A). Taken together, for the first 141 time we filmed assembly and disassembly of T3SS translocons in living host cells, thus providing new 142 insights into the spatiotemporal dynamics of this central T3SS activity.

144 Spatiotemporal dynamics and sequence of PIP2 accumulation and translocon formation in Yersinia- 
146 The ability to film the formation of translocons allowed us to study their spatiotemporal correlation with

147 PIP2 accumulation in Yersinia-containing pre-phagosomes [14, 27]. For this, we infected HeLa cells 148 expressing the PIP2 sensor PLC81-PH-GFP with WA-314 YopD-ALFA in the presence of NbALFA-580 and

149 performed live cell imaging (one z-stack every 20 s). Still frames of a representative event are depicted in

150 Fig. 3C, showing a bacterium being completely enclosed with PIP2 positive host membranes.

151 Recruitment of PLC81-PH-GFP started at one pole of the bacterial cell und then continued until the

152 whole cell was encompassed (Fig. 3C). In the representative example, the time to complete

153 encompassment of the bacterial cell was around $100 \mathrm{~s}$ (start and completion of recruitment at $20 \mathrm{~s}$ and

$154120 \mathrm{~s}$, respectively; Fig. 3C). In this example the first YopD-ALFA signal (at $80 \mathrm{~s}$; Fig. 3C) was observed

155 about $60 \mathrm{~s}$ after the first PLC81-PH-GFP signal and occurred at the pole of the bacterium that was

156 engulfed first by PLC81-PH-GFP (Fig. 3C and D). Thereafter, the number and intensity of the YopD-ALFA

157 translocon signals further increased until the $240 \mathrm{~s}$ time point (Fig. $3 \mathrm{C}$ and D; movie S2). The median time

158 lag between the first visible PLC81-PH-GFP and YopD-ALFA signals was determined to be $2.0+/-4.6 \mathrm{~min}$

159 (median +/- S.D., Fig. 3E). Importantly, in all cases examined, the YopD-ALFA translocon signals occurred

160 after or at the earliest simultaneously with the accumulation of PLCס1-PH-GFP around the bacteria (Fig.

$1613 \mathrm{EE})$. To test the spatiotemporal coordination of PIP2 accumulation and translocon formation in

162 physiological target cells of pathogenic yersiniae, we employed primary human macrophages [28]. Live

163 macrophages expressing PLC81-PH-GFP were infected with WA-314 YopD-ALFA in the presence of

164 NbALFA-580 and investigated with live cell imaging. A representative movie shows that also in the

165 macrophages the bacteria were enclosed with PLC81-PH-GFP positive membrane (at 5 min in Fig. 3F;

166 Movie S3) before the YopD-ALFA translocon signals appeared (at $15 \mathrm{~min}$ in Fig. 3F; Movie S3). In

167 summary, these data demonstrate a close spatiotemporal sequence of PIP2 accumulation and T3SS

168 translocon formation in pre-phagosomes, strongly suggesting that translocon formation requires

169 phosphatidylinositol reorganization in pre-phagosomes. 
171 Spatiotemporal dynamics of galectin-3 and GBP-1 recruitment to Yersinia containing phagosomes

172 harboring translocons

173 It has previously been shown that the T3SS of Yersinia can damage the phagosome membranes

174 surrounding these bacteria in cells $[29,30]$. Yet, the dynamics and spatiotemporal relationship between

175 membrane damage and formation of translocons/pores, that are thought to induce the membrane

176 disruption, have not been elucidated. GFP-galectin-3 has been used as a sensor for membrane damage

177 because of its ability to attach to $\beta$-galactose-containing glycoconjugates present in the luminal leaflet of

178 phagosomal membranes (Fig. 4A) [31]. When expressed in the cytosol of host cells, GFP-galectin-3

179 accumulates at phagosomal membranes when these have been ruptured e.g., by T3SS translocons (Fig.

180 4A). To first confirm that membranes are disrupted by the Yersinia T3SS, we infected HeLa cells

181 expressing GFP-galectin-3 with different Yersinia strains (Table 1). We observed recruitment of GFP-

182 galectin-3 by approximately $13 \%$ of both, cell-associated wild type WA-314 YopD-ALFA and effector-

183 deficient WA-C pTTSS, but not by T3SS-deficient WA-C bacteria (Fig. 4B). This confirms that phagosome

184 disruption is caused by the Yersinia T3SS without the involvement of effectors.

185 To test the spatiotemporal relation of translocon formation and membrane damage, we infected HeLa

186 cells expressing GFP-galectin-3 with WA-314 YopD-ALFA in the presence of NbALFA-580 and performed

187 live cell imaging. This revealed that GFP-galectin-3 recruitment was preceded by detectable YopD-ALFA

188 signals in $92.6 \%+/-5.6 \%$ (mean +/- S.D.) of all GFP-galectin-3 recruitment events (Fig. 4C). The mean

189 time interval between translocon formation and GFP-galectin-3 recruitment was determined to be 13.9

$190+$ +-6.3 min (mean +/- S.D., Fig. 4D). We also noticed that GFP-galectin-3 was recruited relatively abruptly

191 to the entire phagosomal membrane and that its recruitment was accompanied by a decrease in the

192 YopD-ALFA translocon signal ( $n=4$; Fig. 4E and F and movie S4). This suggests that galectin-3 may engage

193 host defense mechanisms eventually leading to degradation of translocon proteins.

194 Recently it was shown that galectin-3 promotes recruitment of the guanylate-binding proteins (GBPs)

195 GBP-1 and GBP-2 to Yersinia-containing compartments [29]. GBPs belong to the family of interferon- 
inducible GTPases and facilitate cell-intrinsic immunity by targeting host defense proteins to pathogen

197 containing compartments [32]. Further, GBP-1 was recently shown to directly bind to LPS of Gram-

198 negative bacteria and function as an LPS-clustering surfactant that disrupts the physicochemical

199 properties of the LPS layer [33-35]. To investigate the spatiotemporal coordination of galectin-3 and

200 GBP-1 recruitment to bacteria that formed translocons, HeLa cells co-expressing galectin-3-mScarlet and

201 GFP-GBP-1 were infected with WA-314 YopD-ALFA in the presence of NbALFA-580 and subjected to live

202 cell imaging (Fig. 4G; Movie S5). GFP-GBP-1 was recruited specifically to bacteria that previously had

203 formed translocons and recruited GFP-galectin-3 (100 \% of observed GFP-GBP-1 recruitment events, $\mathrm{n}=$

204 45). GFP-GBP-1 recruitment (to bacteria that had formed translocons) was regularly observed shortly

205 after or concomitant with GFP-galectin-3 recruitment (Fig. 4G; Movie S5). Staining with an anti-O8 LPS

206 antibody failed to stain GBP-1 coated bacteria suggesting that GBP-1 directly interacts with the Yersinia

207 LPS and thereby hinders detection by the anti-LPS antibody (Fig. 4H). These data suggest that Yersinia

208 translocons cause disruption of phagosomal membranes which leads to sequential galectin-3 and GBP-1

209 recruitment and, likely through the recruitment of additional factors, to translocon degradation.

\section{Discussion}

212 Here we used live cell imaging to characterize the spatiotemporal sequence of molecular events

213 associated with Yersinia translocon assembly and disassembly in host cells. To this end, we constructed a

214 Yersinia strain expressing an ALFA-tag labeled YopD that retained its functionality and could be visualized

215 in fixed and living cells by binding to a fluorescently labeled nanobody (NbALFA). Live cell imaging was

216 also facilitated by the fact that Yersinia translocon formation occurs in a pre-phagosomal host cell

217 compartment where YopD-ALFA is accessible by externally added NbALFA. Thus the incorporation of

218 YopD-ALFA into translocons, as measure for translocon formation, could be recorded and temporally and

219 spatially correlated with the accumulation of the following biosensors i) PLC 1 1-PH-GFP, which senses 
220 PIP2 at the pre-phagosome; ii) GFP-galectin-3, which senses phagosomal membrane disruption; and iii)

221 GFP-GBP1, which senses activation of the cell autonomous immune system at the phagosome.

222 Translocon formation was always initiated seconds to a few minutes after PIP2 accumulation, suggesting

223 that a specific phospholipid composition in the pre-phagosomes may trigger the secretion of translocon

224 proteins by the T3SS and/or is required for their membrane integration. PIP2 rich pre-phagosomes may

225 also recruit host cell receptors like FPR1 and CCR5, which have been reported to promote $Y$. pestis and $Y$.

226 pseudotuberculosis translocon formation [7, 36].

227 Disruption of the phagosomal membrane by Yersinia required a functional T3SS, was associated with

228 only a small fraction of cell-associated bacteria on which we previously detected translocons in $>90 \%$,

229 and occurred with a delay of approximately $14 \mathrm{~min}$ after translocon formation. This indicates that

230 membrane integration of the translocons per se is not sufficient for membrane disruption and

231 subsequent entry of galectin-3. It remains to be elucidated how translocons compromise the phagosome

232 membrane, e.g., whether they act in terms of unregulated translocon activity if they are separated from

233 the T3SS during phagosome maturation or whether membrane disrupting host immune factors that

234 recognize the translocon might be involved.

235 In a recent study galectin-3 was found to recruit the guanylate binding proteins (GBPs) GBP-1 and GBP-2

236 to Yersinia containing vacuoles dependent on a functional T3SS [29]. GBPs belong to the large group of

237 interferon induced antimicrobial host cell factors known to be recruited to pathogen-containing vacuoles

238 (PVs). They escort antimicrobial factors to the PVs and thereby contribute to the cell-autonomous

239 immunity $[32,37]$. Of note, the galectin-3 signal in our study usually covered the whole circumference of

240 the bacteria indicating that the membrane is not substantially ruptured or detached from the bacteria as

241 described for Shigella [31, 38]. GFP-galectin-3 and GBP1-GFP were sequentially recruited to bacteria-

242 containing phagosomes that previously had formed translocons and were associated with a reduction of 
243 the YopD-ALFA and LPS signals. The exact mechanisms responsible for the obvious dissolution of the

244 translocon and the bacterial cell membranes are not known.

245 In summary, we describe here a new method for visualizing and filming the assembly and disassembly of

246 Yersinia translocons by fluorescence microscopy in living cells. In this way, key aspects of the dynamics of

247 translocon formation, its effects on membrane integrity, and recognition by host cell defense

248 mechanisms could be recorded with high spatiotemporal resolution. The described approach might also

249 be valuable for imaging of translocator proteins in other bacterial species. For this it will be critical to

250 insert the tag into positions in the different translocators so that no interference with translocon

251 function occurs.

252 More highly resolved molecular details of T3SS translocon formation, the effects of translocon pores on

253 host membranes and their recognition by host immune factors may become available through the

254 development and application of super resolution live imaging technologies like live cell STED and

255 MINFLUX microscopy [39].

257 Acknowledgements

258 This study was supported by the Joachim Herz Foundation given to Alexander Carsten. We thank the UKE

259 microscopy imaging facility (umif) for training and support. We thank Tomas Edgren for helpful

260 discussion and knowing where to tag YopD. 
264 All standard laboratory chemicals and supplies were purchased from Roth (Karlsruhe, Germany), Sigma-

265 Aldrich (Steinheim, Germany) or Merck (Hohenbrunn, Germany) unless indicated otherwise.

\section{Plasmids}

268 The following plasmids were described previously: PLC81-PH-GFP [40] was provided by T. Balla (National 269 Institutes of Health, Bethesda, MD). The myc-Rac1Q61L plasmid [41] was kindly provided by Dr. Pontus 270 Aspenström (Uppsala University, Uppsala, Sweden) and the GFP-GBP-1 plasmid [35] by P. Broz 271 (University of Lausanne, Epalinges, Switzerland). pEGFP-galectin-3 [42] was purchased from Addgene $272(\# 73080)$ and the mScarlet-galectin 3 was generated by using the pEGFP-galectin-3 plasmid and replacing 273 eGFP by mScarlet at the Nhel/Bglll sites. pCMV-NbALFA-mScarlet-I (NanoTag Biotechnologies, Germany) 274 was used as PCR amplification template (mScarlet fwd Nhel:

TGCCATAGATCTCTTGTACAGCTCGTCCAT). The plasmid construct pMK-bla [43] was kindly provided by Biochemistry, Munich, Germany).

\section{Antibodies and nanobodies}

282 Polyclonal rabbit anti-YopB (aa 1-168) and anti-YopD (aa 150-287) as well as rat anti-YopB (aa 1-168)

283 antibodies were produced as described previously [14]. Rabbit polyclonal anti-Y. enterocolitica 0:8 was

284 purchased from Sifin (Berlin, Germany). Secondary anti-IgG antibodies and their sources were: Alexa488

285 chicken goat anti-rat, Alexa568 goat anti-rabbit, Alexa647 goat anti-rabbit, (Molecular Probes, Karlsruhe,

286 Germany), horseradish peroxidase linked donkey anti-rabbit (GE Healthcare, Chicago, USA).

287 Fluorescently labeled primary camelid anti-ALFA nanobodies (NbALFA) and their source were: Alexa 


\begin{tabular}{|l|l|}
\hline YopD-ALFA HomA fwd & $\begin{array}{l}\text { TATTATCCTAACTTATTATTTTTAATTTAATAATAAAAGCCCTGGATTACCA } \\
\text { TTAGTTAA }\end{array}$ \\
\hline YopD-ALFA HomA rev & $\begin{array}{l}\text { TTGGAAGAGGAACTGAGACGCCGCTTAACTGAACCAGGCGGAGGTGGAT } \\
\text { CTATCGGGAGAATATGGAAACCAGA }\end{array}$ \\
\hline YopD-ALFA HomB fwd & $\begin{array}{l}\text { GCGGCGTCTCAGTTCCTCTTCCAAACGGCTCGGGCCACCAGACCCGCCCG } \\
\text { AACCACCATCCTCTCTGCTTACCGCTTTAT }\end{array}$ \\
\hline YopD-ALFA HomB rev & AAAGCGGTGAGGTTAAAAAAA \\
\hline YopD-crRNA fwd & TAGATCATATTCTCCCGATATCCTC \\
\hline YopD-crRNA rev & AGACGAGGATATCGGGAGAATATGA \\
\hline $\begin{array}{l}\text { final insert sequence } \\
\text { (linker, }\end{array}$ & ALFA-tag, linker)
\end{tabular}

Approval for the analysis of anonymized blood donations (WF-015/12) was obtained by the Ethical Committee of the Ärztekammer Hamburg (Germany).

\section{Source and generation of Yersinia mutants}

The Yersinia strains used here are listed in Table 1. Y. enterocolitica wild type strain WA-314 was a gift of amplified from the $Y$. enterocolitica pYV virulence plasmid with the reverse primer YopD-ALFA HomA rev 
including the remaining part of the ALFA-tag insert and linker and the corresponding reverse primer

YopD-ALFA HomB rev. Both homology arms were used as templates in an overlap extension PCR using

the outer primers (YopD-ALFA HomA fwd and YopD-ALFA HomB rev) to generate the final HDR fragment.

The crRNAs required for targeting Cas12a to the defined insertion site were designed based on the $20 \mathrm{bp}$

protospacer following the $3^{\prime}$-end of a PAM $\left(5^{\prime}-\mathrm{TTN}-3^{\prime}\right)$. The respective oligonucleotides were designed

with Eco31L overhangs at the 5'- and 3'-ends (YopD-crRNA fwd and YopD-crRNA rev), annealed and

312 ligated into the Eco31L digested pAC-crRNA vector harboring also a sacB sucrose sensitivity gene. $700 \mathrm{ng}$

of the HDR fragment and $350 \mathrm{ng}$ of the PAC-crRNA were electroporated into an electrocompetent WA-

314 strain carrying pKD46-Cas12a, which harbors the lambda Red recombinase under control of an

arabinose inducible promotor, Cas12a (Cas12a/Cpf1 from Francisella novicida) and a temperature-

Table 1:

\begin{tabular}{|l|l|l|}
\hline Strain & Relevant characteristic & Source/References \\
\hline WA-314 & $\begin{array}{l}\text { wild type strain carrying } \\
\text { virulence plasmid pYV; } \\
\text { serogroup O8; kanamycin } \\
\text { resistance cassette in non- } \\
\text { coding region of pYV-O8 }\end{array}$ & {$[45,47]$} \\
\hline WA-C & pYV-cured derivative of WA-314 & {$[47]$} \\
\hline WA-C pTTSS & $\begin{array}{l}\text { WA-C harboring pTTSS encoding } \\
\text { the TTSS secretion/translocation } \\
\text { apparatus of WA-314 but no } \\
\text { Yop effector genes; SptR }\end{array}$ & {$[48]$} \\
\hline WA-314DYopD & $\begin{array}{l}\text { WA-C harboring pYVDyopD; } \\
\text { KanR }\end{array}$ & {$[14]$} \\
\hline WA-314 YopD-ALFA & $\begin{array}{l}\text { WA-314 with ALFA-tag inserted } \\
\text { in YopD; KanR }\end{array}$ & this study \\
\hline WA-314 pMK-bla & $\begin{array}{l}\text { WA-314 harboring pMK-bla } \\
\text { containing YopE53- } \beta \text {-lactamase } \\
\text { fusion; Kan }{ }^{\mathrm{R}} \text {, CM }\end{array}$ & {$[25]$} \\
\hline
\end{tabular}




\begin{tabular}{|l|l|l|}
\hline WA-314 YopD-ALFA pMK-bla & $\begin{array}{l}\text { WA-314 YopD-ALFA harboring } \\
\text { pYopE-bla; } \operatorname{Kan}^{\mathrm{R}}, \mathrm{CM}^{\mathrm{R}}\end{array}$ & this study \\
\hline
\end{tabular}

\section{Cell culture and transfection}

323 HeLa cells (ACC\#57, DSMZ-German Collection of Microorganisms and Cell Cultures) were cultured at $37^{\circ} \mathrm{C}$ 324 and 5\% CO2 in DMEM (Invitrogen, GIBCO, Darmstadt, Germany) supplemented with 10\% FCS (v/v). For 325 infection with bacteria, HeLa cells were seeded in 6 well plates ( $3 \times 10^{5}$ cells per well) or on glass 326 coverslips (6x104 cells per well; confocal: Precision coverslips, round, $12 \mathrm{~mm}$ diameter, No 1.5, with 327 precision thickness, Hartenstein, Würzburg, Germany; STED: $12 \mathrm{~mm}$, No. $1.5 \mathrm{H}$ for high resolution, 328 Marienfeld $\mathrm{GmbH}$, Lauda-Königshafen, Germany). For live imaging $2.5 \times 10^{4}$ HeLa cells were seeded in 329 ibidi $\mu$-slide 8 wells (ibidi, Martinsried, Germany). HeLa cells were transfected with $0.25 \mu \mathrm{g}$ plasmid for 330 coverslips or $0.125 \mu \mathrm{g}$ plasmid for 8 well slides using Turbofect (Thermo Fisher Scientific, Waltham, 331 Massachusetts, USA) for $16 \mathrm{~h}$ according to the manufacturer's protocol.

332 Human peripheral blood monocytes were isolated from heparinized blood as described previously [77].

333 Monocytes/Macrophages were cultured in RPMI1640 (Invitrogen) containing $20 \%$ heterologous human 334 serum (v/v) for 7 days with medium changes every three days. Macrophages were transfected with the 335 Neon Transfection System (Invitrogen) with $5 \mu$ g DNA per $10^{6}$ cells (1000 V, 40 ms, 2 pulses) and infected $3364 \mathrm{~h}$ after transfection.

\section{Preparation of bacteria}

339 Yersinia were grown in Luria Bertani (LB) broth supplemented with nalidixic acid, kanamycin, 340 spectinomycin or chloramphenicol as required at $27^{\circ} \mathrm{C}$ overnight and then diluted 1:20 in fresh LB broth, 341 followed by cultivation at $37^{\circ} \mathrm{C}$ for $1.5 \mathrm{~h}$ to induce expression of the T3SS. For cell infection, bacteria 342 were centrifuged, resuspended in ice-cold PBS and added to target cells at a defined multiplicity of 343 infection (MOI), as specified in the figure captions. Bacteria were then centrifuged at $200 \times g$ for $1 \mathrm{~min}$ onto the target cells to synchronize the bacterial attachment. For in-vitro Yop secretion, EGTA (5 mM), 
$345 \mathrm{MgCl} 2(15 \mathrm{mM})$ and glucose $(0.2 \%, \mathrm{w} / \mathrm{v})$ was added to the growth medium for $\mathrm{Ca} 2+$ chelation after $1.5 \mathrm{~h}$

346 at $37^{\circ} \mathrm{C}$, followed by another $3 \mathrm{~h}$ of incubation at $37^{\circ} \mathrm{C}$, as described before [48]. The resulting samples

347 were analyzed by SDS-PAGE, followed by either Coomassie staining or transfer to a PVDF membrane

348 (Immobilon-P, Millipore), and analysis by Western blot using antisera against YopB and YopD.

\section{$350 \quad$ Fluorescence labeling}

351 Infected cells were washed twice with PBS and fixed with 4\% PFA (v/v; Electron Microscopy Science,

352 Hatfield, USA) in PBS for 5 min. Samples were treated with digitonin solution (90 $\mu \mathrm{g} / \mathrm{mL}$ in PBS) to

353 permeabilize cellular membranes and allow access of the nanobody to translocon-associated YopD-ALFA.

354 For antibody stainings or staining of intrabacterial YopD-ALFA using the nanobody, samples were

355 permeabilized with $0.1 \%$ Triton $\mathrm{X}-100(\mathrm{v} / \mathrm{v})$ in PBS for $15 \mathrm{~min}$. After fixation and permeabilization

356 coverslips were washed twice with PBS. Unspecific binding sites were blocked with 3\% bovine serum

357 albumin (BSA, w/v) in PBS for at least $30 \mathrm{~min}$. Samples were then incubated with the indicated primary

358 antibody (1:50) or fluorescently labeled FluoTag ${ }^{\circledR}-\mathrm{X} 2$ anti-ALFA nanobody (1:200) for $1 \mathrm{~h}$ (16 h for STED

359 samples using the nanobody) and incubated with a 1:200 dilution of the suitable fluorophore-coupled

360 secondary antibody or fluorophore-coupled phalloidin (1:200, Invitrogen) and 4',6-diamidino-2-

361 phenylindole (DAPI; $300 \mathrm{nM}$, Invitrogen) as indicated for $45 \mathrm{~min}$. Nanobodies as well as primary and

362 secondary antibodies were applied in PBS supplemented with 3\% BSA. After each staining coverslips

363 were washed three times with PBS. Coverslips for confocal microscopy were mounted in ProLong

364 Diamond (Thermo Fisher Scientific) while STED samples were mounted in ProLong Gold (Thermo Fisher

365 Scientific, Waltham, USA). 
368 Fixed samples were analyzed with a confocal laser scanning microscope (Leica TCS SP8) equipped with a

$36963 x$ oil immersion objective (NA 1.4) and Leica LAS X SP8 software (Leica Microsystems, Wetzlar, 370 Germany) was used for acquisition.

$372 \quad$ Live cell imaging

373 For live imaging the cells were 8 well slides were placed in the prewarmed chamber supplied with $5 \%$

374 CO2 of the spinning disc microscope Visitron SD-TRIF (Nikon Eclipse TiE, Nikon, Japan) with a 63x oil

375 immersion objective (NA 1.40) and the VisiView software (Visitron Systems, Germany). The nanobody

376 was diluted 1:300 in $200 \mu$ I DMEM with $10 \%$ FCS and mixed with the WA-314 YopD-ALFA. The number of

377 bacteria was chosen according to the intended MOI. The medium was removed from the cells and the

$378200 \mu \mathrm{l}$ medium containing nanobody and bacteria was added. The imaging process was started

379 immediately.

\section{Super resolution imaging}

382 STED nanoscopy and corresponding confocal microscopy were carried out in line sequential mode using 383 an Abberior Instruments Expert Line STED microscope based on a Nikon Ti-E microscopy body and 384 employed for excitation and detection of the fluorescence signal a 60x Plan APO 1.4 oil immersion objective. A pulsed $640 \mathrm{~nm}$ laser was used for excitation and a pulsed near-infrared laser $(775 \mathrm{~nm})$ was used for STED. The detected fluorescence signal was directed through a variable sized pinhole (1 Airy unit at $640 \mathrm{~nm}$ ) and detected by avalanche photo diodes (APDs) with appropriate filter settings for Cy5 (615 - $755 \mathrm{~nm}$ ). Images were recorded with a dwell time of $0.5 \mu$ s and the pixel size was set to be $10 \mathrm{~nm}$. of 8 ns. STED images were acquired with a 2D-STED donut. 
393 The z-stacks of images acquired of both fixed and live samples were combined to one image using 394 maximum intensity projection. These images were used to determine the lifespan of a detectable 395 translocon signal, the fluorescence intensity of PLCD1-PH and YopD-ALFA signal in a region of interest 396 around the bacterium for each time point as well as the time PLC $1-\mathrm{PH}$ is present before translocon 397 formation. In addition, the percentage of galectin-3 positive bacteria in cells harboring translocon 398 forming bacteria was quantified in fixed samples. Live imaging was used to determine the number of 399 galectin-3 positive bacteria with and without prior YopD-ALFA signal as well as the time interval between 400 translocon formation and galectin-3 recruitment. The fluorescence intensity of YopD-ALFA and GFP401 galectin-3 signal was measured in a region of interest around the bacterium for each time point of a 402 representative movie. In general, the fluorescence intensity measurements were normalized to the 403 lowest intensity measured and the highest intensity value was set to $100 \%$. Live imaging data were 404 additionally analyzed for the presence of galectin-3 before GBP-1 recruitment to phagosomes.

\section{Detection of Bla-activity by immunofluorescence microscopy}

407 One day before infection $2.5 \times 10^{4} \mathrm{HeLa}$ cells were seeded in ibidi $\mu$-Slide 8 wells. The following day cells 408 were infected with different bacterial strains as indicated and after 30 min the medium was replaced by $409200 \mu \mathrm{l} \mathrm{CCF} 4$ /AM loading solution (prepared according to the manufacturer's instructions) diluted in 410 DMEM supplemented with 10\% FCS and $2.5 \mathrm{mM}$ probenecid. The cells were placed in the prewarmed 411 chamber supplied with 5\% CO2 of the laser scanning microscope Leica TCS SP8 and imaging was 412 performed using a 20x oil immersion objective (NA 0.75) and the Leica LAS X SP8 software (Leica 413 Microsystems, Wetzlar, Germany). 
Fig. 1: Insertion of the ALFA-tag into YopD does not interfere with protein function and allows for nanobody-based staining of translocons. adapted from [16] and based on data on interactions of Pseudomonas aeruginosa PopD and PopB. The red box indicates the inserted ALFA-tag between amino acids 194 and 195 on the extracellular part of permeabilized with digitonin. Co-staining of translocon components was conducted with anti-YopB (shown in green) and anti-YopD (shown in red) antibodies and NbALFA-635 (shown in magenta). Scale with a cell permeant FRET dye (CCF4/AM) were infected for $1 \mathrm{~h}$ with WA-314, WA-314 pYopE-bla and coumarin results in FRET to fluorescein in the uncleaved CCF4 emitting a green fluorescent signal. 
441 for WA-314, WA-314 pMK-bla and WA-314-YopD-ALFA pMK-bla, respectively. (F) Cytotoxicity assay.

442 HeLa cells were infected for $1 \mathrm{~h}$ with WA-314, WA-314 YopD-ALFA and WA-314DYopD at an MOI of 100

443 and imaged by phase contrast microscopy. Depicted are phase contrast images of a representative

444 experiment. Scale bar: $20 \mu \mathrm{m}$.

446 Fig. 2: Differential permeabilization for selective staining of YopD-ALFA in different cellular 447 compartments.

448 (A) Selective nanobody staining of YopD-ALFA in different cellular compartments. The schematic (top)

449 shows different levels of host- and bacterial cell permeabilization and according accessibility of different 450 pools of YopD-ALFA for NbALFA staining. Rac1Q61L expressing HeLa cells were infected with WA-314

451 YopD-ALFA at an MOI of 10 for $1 \mathrm{~h}$, fixed and stained with NbALFA-635 without prior permeabilization to 452 specifically target translocon associated YopD-ALFA in the pre-phagosomal compartment (left, shown in 453 red). Host cell membranes were permeabilized with digitonin and translocons located in closed 454 phagosomes were stained with NbALFA-580 (middle, shown in magenta). Note that pre-phagosomal 455 YopD-ALFA was already saturated with NbALFA-635 (red) during the first staining step. Finally, also the 456 bacterial membranes were permeabilized with triton and the intrabacterial pool of YopD-ALFA was 457 stained with NbALFA-488 (right, shown in green). Scale bar: $2 \mu \mathrm{m}$. (B) STED imaging of intrabacterial and 458 translocon-associated YopD-ALFA. Rac1Q61L expressing HeLa cells were infected with WA-314 YopD459 ALFA at an $\mathrm{MOI}$ of 10 for $1 \mathrm{~h}$, fixed and stained with NbALFA-635 (shown in red) with prior 460 permeabilization of host cell membranes using digitonin to target translocon associated YopD-ALFA. 461 Bacterial membranes were permeabilized with triton and the intrabacterial pool of YopD-ALFA was 462 stained with NbALFA-580 (shown in green). The images were acquired using super resolution STED 463 microscopy. The boxed region in the left of the image is depicted as enlargements in separate channels 464 at the side. Scale bar: $1 \mu \mathrm{m}$ (overview) and $200 \mathrm{~nm}$ (enlargements). 
468 (A) Live imaging of translocons during HeLa cell infection. HeLa cells expressing myc-Rac1Q61L and GFPin the cell culture medium. Cells were imaged with a spinning disk microscope recording z-stacks every minute. Stacks for each time point were combined to one image using maximum intensity projection and one image every $5 \mathrm{~min}$ is shown. The left panel shows the overview image at $0 \mathrm{~min}$. The boxed region in the overview image shows the area of the video depicted in still frames to the right. Dashed white lines indicate the outline of the bacteria. Scale bars: $10 \mu \mathrm{m}$ (overview) and $2 \mu \mathrm{m}$ (still frames). (B) Lifespan of

the translocon. The lifespan of the translocons was determined using movies that recorded the YopD$\mathrm{n}=25$ bacteria (7 independent experiments, 13 host cells) (C) PIP2 accumulation at the host membrane were infected with WA-314 YopD-ALFA at an MOI of 20 and incubated with NbALFA-580 diluted in cell culture medium. Cells were imaged with a spinning disk microscope recording z-stacks every 20 s. Stacks

for each time point were combined to one image using maximum intensity projection and one image every $20 \mathrm{~s}$ is shown. The left panel shows the overview image at $0 \mathrm{~min}$. The boxed region in the overview image shows the area of the video depicted in still frames to the right. White arrows indicate the appearance of PLC81-PH-GFP and the first translocon signal. Scale bar: $10 \mu \mathrm{m}$ (overview) and $2 \mu \mathrm{m}$ (still

frames). (D) Fluorescence intensities of PIP2 marker PLC81-PH-GFP and YopD-ALFA signals. The relative 

human macrophages. Primary human macrophages expressing PLC81-PH-GFP were infected with WA314 YopD-ALFA at an MOI of 20 and incubated with NbALFA-580 diluted in cell culture medium. Cells were combined to one image using maximum intensity projection and one image every 5 min is shown.

496 The left panel shows the overview image at $0 \mathrm{~min}$. The boxed region in the overview image shows the 497 area of the video depicted in still frames to the right. White arrows indicate the appearance of PLC $81-\mathrm{PH}-$ GFP and the first translocon signal. Scale bars: $10 \mu \mathrm{m}$ (overview) and $2 \mu \mathrm{m}$ (still frames).

Fig. 4: Nanobody-based live imaging of translocons: Galectin-3 and GBP-1 recruitment upon translocon induced membrane damage.

(A) Schematic representation of galectin-3 recruitment following membrane damage during infection. Galectin-3 (shown in green) is found in the cytosol of the host cell. Translocon formation appears to induce membrane damage allowing access of galectin-3 to glycans in the lumen of vacuoles. (B) Vacuolar membrane damage by the T3SS. HeLa cells expressing myc-Rac1Q61L and GFP-galectin-3 were infected with WA-C, WA-C pTTSS and WA-314 YopD-ALFA at an MOI of 100 for $1 \mathrm{~h}$, fixed and permeabilized using digitonin. Cells were stained with anti-YopD antibody, Alexa633 phalloidin and DAPI. The percentage of galectin-3 positive bacteria per cell was quantified for WA-C, WA-C pTTSS and WA-314 YopD-ALFA ( $n=$ 1680, 9 host cells; $n=508$ bacteria, 7 host cells; $n=1065$ bacteria, 10 host cells). Only cells harboring translocon forming bacteria were analyzed for WA-C pTTSS and WA-314 YopD-ALFA infections. (C)

511 Fraction of galectin recruitments without and with prior YopD-ALFA signal. HeLa cells expressing myc-

512 Rac1Q61L and GFP-galectin-3 were infected with WA-314 YopD-ALFA at an MOI of 20 and incubated with 513 NbALFA-580 diluted in cell culture medium. Cells were imaged with a spinning disk microscope recording 514 z-stacks every minute. Galectin-3 recruitment events were quantified with respect to whether YopD515 ALFA signal is present before recruitment. $\mathrm{n}=330$ uptake events (6 independent experiments, 38 
517 cells expressing myc-Rac1Q61L and GFP-galectin-3 were infected with WA-314 YopD-ALFA at an MOI of 51820 and incubated with NbALFA-580 diluted in cell culture medium. Cells were imaged with a spinning 519 disk microscope recording z-stacks every minute. The time intervals between first occurrence of the 520 translocon signal and first GFP-galectin-3 signals were measured. Each dot represents one measurement.

$521 \mathrm{n}=36$ bacteria (4 independent experiments; 9 movies). (E) Galectin-3 recruitment to phagosomes

522 containing translocon forming bacteria. Live imaging experiments were performed as in (C). The z-stacks

523 for each time point were combined to one image using maximum intensity projection and one image

524 every $5 \mathrm{~min}$ is representatively shown. Scale bar: $2 \mu \mathrm{m}$. The relative fluorescence intensities at the 525 bacteria were plotted to illustrate the temporal relationship of NbALFA-580 and GFP-galectin-3 signals.

526 (F) Loss of YopD-ALFA signal after GFP-galectin-3 recruitment. Live imaging experiments were 527 performed as in (C). The relative fluorescence intensities of the translocon signal were measured in the 528 last frame before and in the frames $5 \mathrm{~min}$ and $10 \mathrm{~min}$ after recruitment of GFP-galectin-3. $\mathrm{n}=4$ measurements (1 experiment, 3 host cells). (G) GBP-1 recruitment to galectin-3 positive bacteria. HeLa 530 cells expressing myc-Rac1Q61L, mScarlet-galectin-3 (shown in green) and GFP-GBP-1 (shown in red) 531 were infected with WA-314 YopD-ALFA at an MOI of 20 and incubated with NbALFA-580 (shown in 532 magenta) diluted in cell culture medium. Cells were imaged with a spinning disk microscope recording z533 stacks every 5 minutes. The z-stacks for each time point were combined to one image using maximum 534 intensity projection and images are shown starting $20 \mathrm{~min}$ after uptake of the bacteria. Scale bar: $5 \mu \mathrm{m}$.

535 (H) GBP-1 positive bacteria lack LPS antibody staining. HeLa cells expressing myc-Rac1Q61L and GFP536 GBP-1 (shown in green) were infected with WA-314 YopD-ALFA at an MOI of 30, fixed and permeabilized 537 using digitonin. Cells were stained with anti-LPS antibody (shown in magenta). The boxed regions (I, II) in 538 the overview image are depicted as enlargements in separate channels to the right. Dashed white lines 539 indicate the outline of the nucleus in the overview image and the bacteria in the enlargements. Scale 540 bar: $10 \mu \mathrm{m}$ (overview) and $2 \mu \mathrm{m}$ (enlargement). 


\section{References}

542 1. Deng, W., et al., Assembly, structure, function and regulation of type III secretion systems. Nat 543 Rev Microbiol, 2017. 15(6): p. 323-337.

$5442 . \quad$ Lara-Tejero, M. and J.E. Galan, The Injectisome, a Complex Nanomachine for Protein Injection into Mammalian Cells. EcoSal Plus, 2019. 8(2).

3. Pinaud, L., P.J. Sansonetti, and A. Phalipon, Host Cell Targeting by Enteropathogenic Bacteria T3SS Effectors. Trends Microbiol, 2018. 26(4): p. 266-283.

4. Kubori, T., et al., Supramolecular structure of the Salmonella typhimurium type III protein secretion system. Science, 1998. 280(5363): p. 602-5.

5. Schraidt, O. and T.C. Marlovits, Three-dimensional model of Salmonella's needle complex at subnanometer resolution. Science, 2011. 331(6021): p. 1192-5.

6. Mueller, C.A., et al., The V-antigen of Yersinia forms a distinct structure at the tip of injectisome needles. Science, 2005. 310(5748): p. 674-6.

7. Osei-Owusu, P., et al., FPR1 is the plague receptor on host immune cells. Nature, 2019. 574(7776): p. 57-62.

8. Mattei, P.J., et al., Membrane targeting and pore formation by the type III secretion system translocon. FEBS J, 2011. 278(3): p. 414-26.

9. Mueller, C.A., P. Broz, and G.R. Cornelis, The type III secretion system tip complex and translocon. Mol Microbiol, 2008. 68(5): p. 1085-95.

10. Neyt, C. and G.R. Cornelis, Insertion of a Yop translocation pore into the macrophage plasma membrane by Yersinia enterocolitica: requirement for translocators YopB and YopD, but not LcrG. Mol Microbiol, 1999. 33(5): p. 971-81.

11. Ide, T., et al., Characterization of translocation pores inserted into plasma membranes by type IIIsecreted Esp proteins of enteropathogenic Escherichia coli. Cell Microbiol, 2001. 3(10): p. 669-79.

12. Blocker, A., et al., The tripartite type III secreton of Shigella flexneri inserts IpaB and IpaC into host membranes. J Cell Biol, 1999. 147(3): p. 683-93.

13. Park, D., et al., Visualization of the type III secretion mediated Salmonella-host cell interface using cryo-electron tomography. Elife, 2018. 7.

14. Nauth, T., et al., Visualization of translocons in Yersinia type III protein secretion machines during host cell infection. PLoS Pathog, 2018. 14(12): p. e1007527.

15. Yao, Z. and R. Carballido-Lopez, Fluorescence imaging for bacterial cell biology: from localization to dynamics, from ensembles to single molecules. Annu Rev Microbiol, 2014. 68: p. 459-76.

16. Armentrout, E.I. and A. Rietsch, The Type III Secretion Translocation Pore Senses Host Cell Contact. PLoS Pathog, 2016. 12(3): p. e1005530.

17. Radics, J., L. Konigsmaier, and T.C. Marlovits, Structure of a pathogenic type 3 secretion system in action. Nat Struct Mol Biol, 2014. 21(1): p. 82-7.

18. Gawthorne, J.A., et al., Visualizing the Translocation and Localization of Bacterial Type III Effector Proteins by Using a Genetically Encoded Reporter System. Appl Environ Microbiol, 2016. 82(9): p. 2700-2708.

19. Goser, V., et al., Self-Labeling Enzyme Tags for Analyses of Translocation of Type III Secretion System Effector Proteins. mBio, 2019. 10(3).

20. Van Engelenburg, S.B. and A.E. Palmer, Imaging type-III secretion reveals dynamics and spatial segregation of Salmonella effectors. Nat Methods, 2010. 7(4): p. 325-30.

21. Enninga, J., et al., Secretion of type III effectors into host cells in real time. Nat Methods, 2005. 2(12): p. 959-65.

22. Ehsani, S., C.D. Rodrigues, and J. Enninga, Turning on the spotlight--using light to monitor and characterize bacterial effector secretion and translocation. Curr Opin Microbiol, 2009. 12(1): p. 24-30. 
589

590

591

592

593

594

595

596

597

598

599

600

601

602

603

604

605

606

607

608

609

610

611

612

613

614

615

616

617

618

619

620

621

622

623

624

625

626

627

628

629

630

631

632

633

634

635

636

23. Gotzke, H., et al., The ALFA-tag is a highly versatile tool for nanobody-based bioscience applications. Nat Commun, 2019. 10(1): p. 4403.

24. Charpentier, X. and E. Oswald, Identification of the secretion and translocation domain of the enteropathogenic and enterohemorrhagic Escherichia coli effector Cif, using TEM-1 betalactamase as a new fluorescence-based reporter. J Bacteriol, 2004. 186(16): p. 5486-95.

25. Wolters, M., et al., Cytotoxic necrotizing factor- $Y$ boosts Yersinia effector translocation by activating Rac protein. J Biol Chem, 2013. 288(32): p. 23543-53.

26. Wolters, M., et al., Analysis of Yersinia enterocolitica Effector Translocation into Host Cells Using Beta-lactamase Effector Fusions. J Vis Exp, 2015(104).

27. Sarantis, H., et al., Yersinia entry into host cells requires Rab5-dependent dephosphorylation of PI(4,5)P(2) and membrane scission. Cell Host Microbe, 2012. 11(2): p. 117-28.

28. Bekere, I., et al., Yersinia remodels epigenetic histone modifications in human macrophages. PLoS Pathog, 2021. 17(11): p. e1010074.

29. Feeley, E.M., et al., Galectin-3 directs antimicrobial guanylate binding proteins to vacuoles furnished with bacterial secretion systems. Proc Natl Acad Sci U S A, 2017. 114(9): p. E1698E1706.

30. Zwack, E.E., et al., Guanylate Binding Proteins Regulate Inflammasome Activation in Response to Hyperinjected Yersinia Translocon Components. Infect Immun, 2017. 85(10).

31. Paz, I., et al., Galectin-3, a marker for vacuole lysis by invasive pathogens. Cell Microbiol, 2010. 12(4): p. 530-44.

32. Pilla-Moffett, D., et al., Interferon-Inducible GTPases in Host Resistance, Inflammation and Disease. J Mol Biol, 2016. 428(17): p. 3495-513.

33. Kutsch, M. and J. Coers, Human guanylate binding proteins: nanomachines orchestrating host defense. FEBS J, 2021. 288(20): p. 5826-5849.

34. Kutsch, M., et al., Direct binding of polymeric GBP1 to LPS disrupts bacterial cell envelope functions. EMBO J, 2020. 39(13): p. e104926.

35. Santos, J.C., et al., Human GBP1 binds LPS to initiate assembly of a caspase-4 activating platform on cytosolic bacteria. Nat Commun, 2020. 11(1): p. 3276.

36. Sheahan, K.L. and R.R. Isberg, Identification of mammalian proteins that collaborate with type III secretion system function: involvement of a chemokine receptor in supporting translocon activity. mBio, 2015. 6(1): p. e02023-14.

37. Kutsch, M. and J. Coers, Human guanylate binding proteins: nanomachines orchestrating host defense. FEBS J, 2020.

38. Chang, Y.Y., et al., Shigella hijacks the exocyst to cluster macropinosomes for efficient vacuolar escape. PLoS Pathog, 2020. 16(8): p. e1008822.

39. Carsten, A., et al., Visualization of bacterial type 3 secretion system components down to the molecular level by MINFLUX nanoscopy. bioRxiv, 2021: p. 2021.09.27.461991.

40. Balla, T. and P. Varnai, Visualization of cellular phosphoinositide pools with GFP-fused proteindomains. Curr Protoc Cell Biol, 2009. Chapter 24: p. Unit 244.

41. Aspenstrom, P., A. Fransson, and J. Saras, Rho GTPases have diverse effects on the organization of the actin filament system. Biochem J, 2004. 377(Pt 2): p. 327-37.

42. Maejima, I., et al., Autophagy sequesters damaged lysosomes to control lysosomal biogenesis and kidney injury. EMBO J, 2013. 32(17): p. 2336-47.

43. Koberle, M., et al., Yersinia enterocolitica targets cells of the innate and adaptive immune system by injection of Yops in a mouse infection model. PLoS Pathog, 2009. 5(8): p. e1000551.

44. Riedl, J., et al., Lifeact: a versatile marker to visualize F-actin. Nat Methods, 2008. 5(7): p. 605-7.

45. Oellerich, M.F., et al., Yersinia enterocolitica infection of mice reveals clonal invasion and abscess formation. Infect Immun, 2007. 75(8): p. 3802-11. 
637 46. Yan, M.Y., et al., CRISPR-Cas12a-Assisted Recombineering in Bacteria. Appl Environ Microbiol, 638 2017. 83(17).

639 47. Heesemann, J. and R. Laufs, Construction of a mobilizable Yersinia enterocolitica virulence

640 plasmid. J Bacteriol, 1983. 155(2): p. 761-7.

641

642

643

48. Trulzsch, K., et al., Analysis of chaperone-dependent Yop secretion/translocation and effector function using a mini-virulence plasmid of Yersinia enterocolitica. Int J Med Microbiol, 2003.

644

645 293(2-3): p. 167-77.

49. Berger, C., et al., Structure of the Yersinia injectisome in intracellular host cell phagosomes revealed by cryo FIB electron tomography. J Struct Biol, 2021. 213(1): p. 107701.

646 


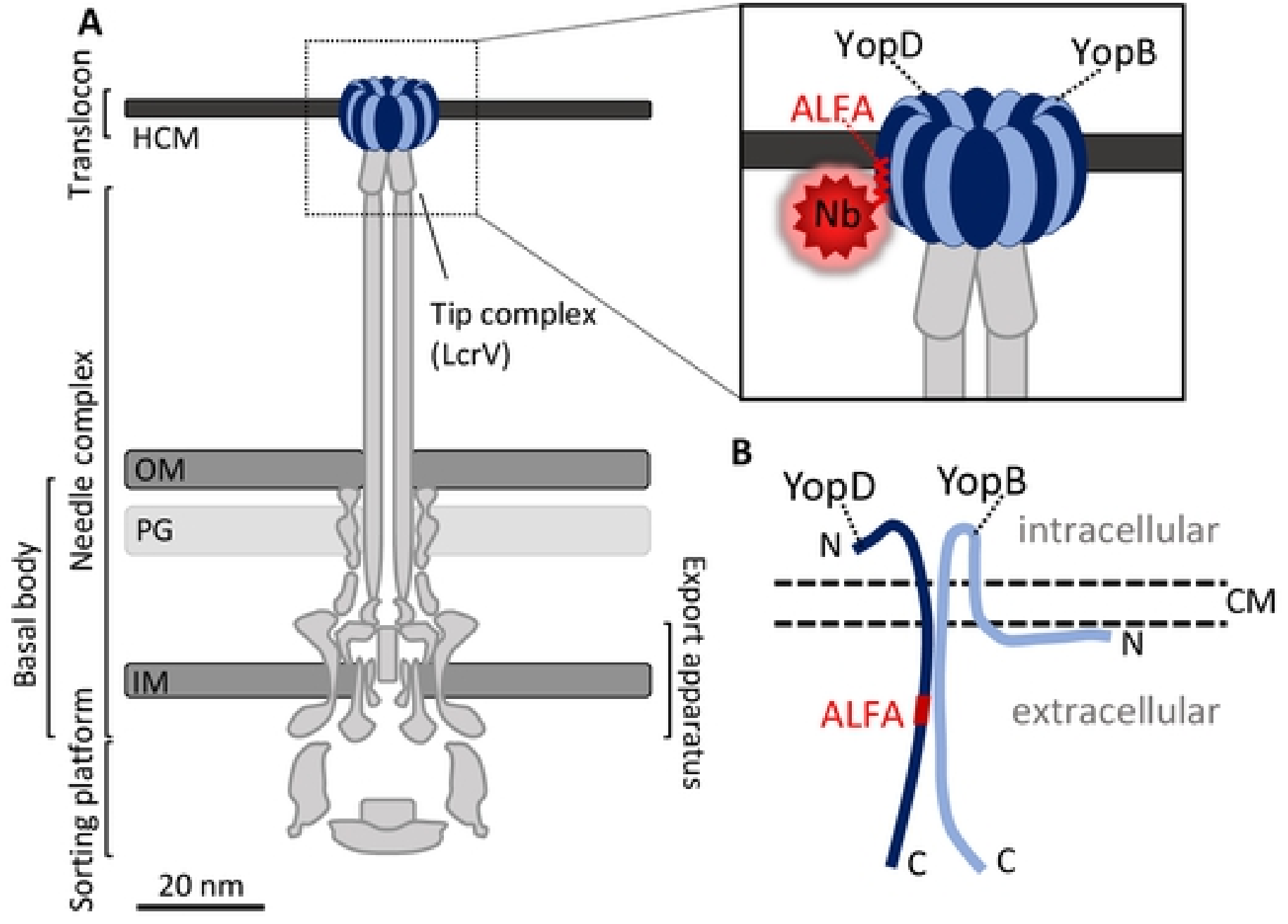

bioRxiv preprint doi: https://doi.org/10 1101/2022 481.10 .475601 his version posted January 10, 2022. The copyright holder for this preprint whichlwas not certified by peer reviewy ts the abtanortunder, what
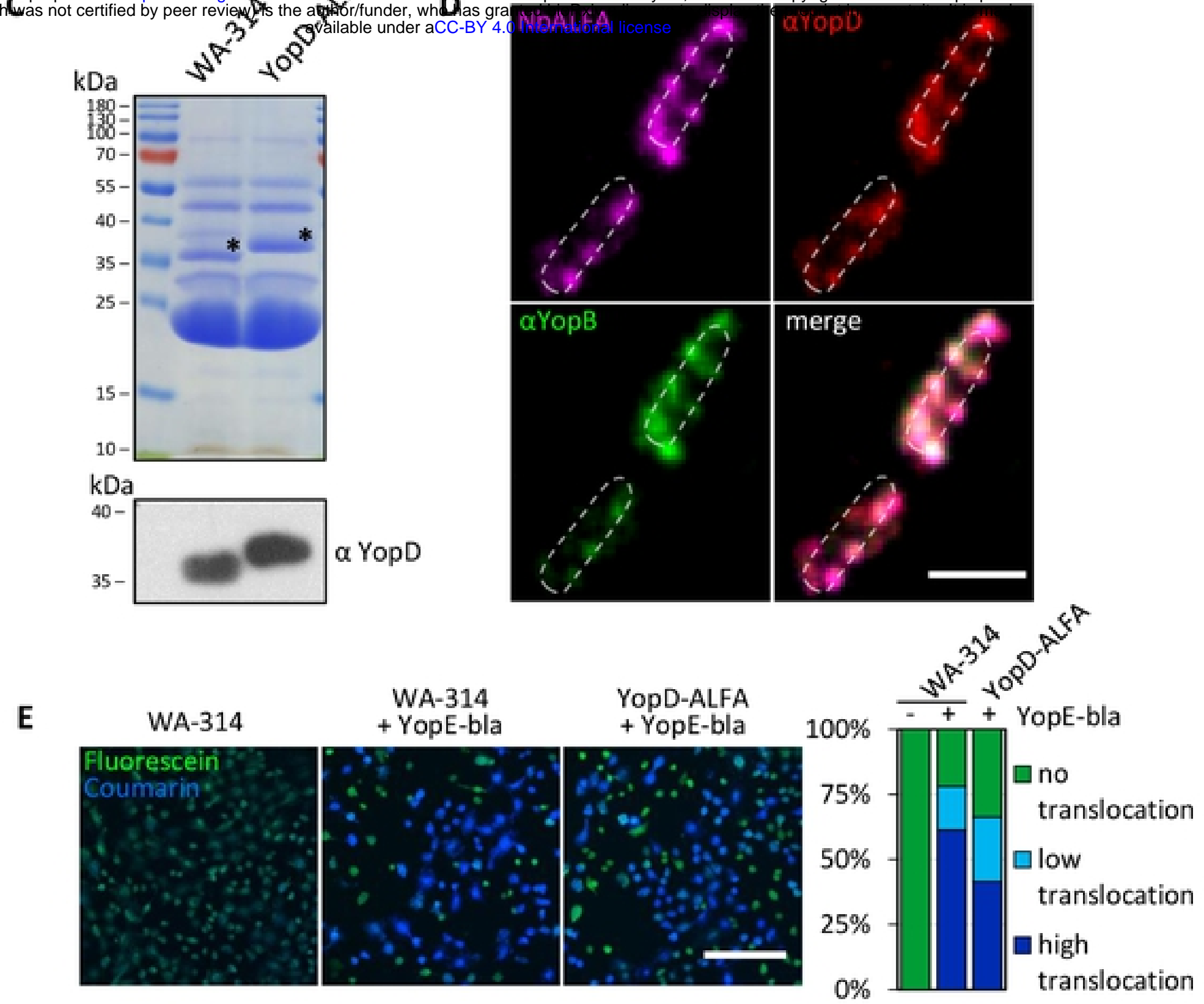

$\mathbf{F}$

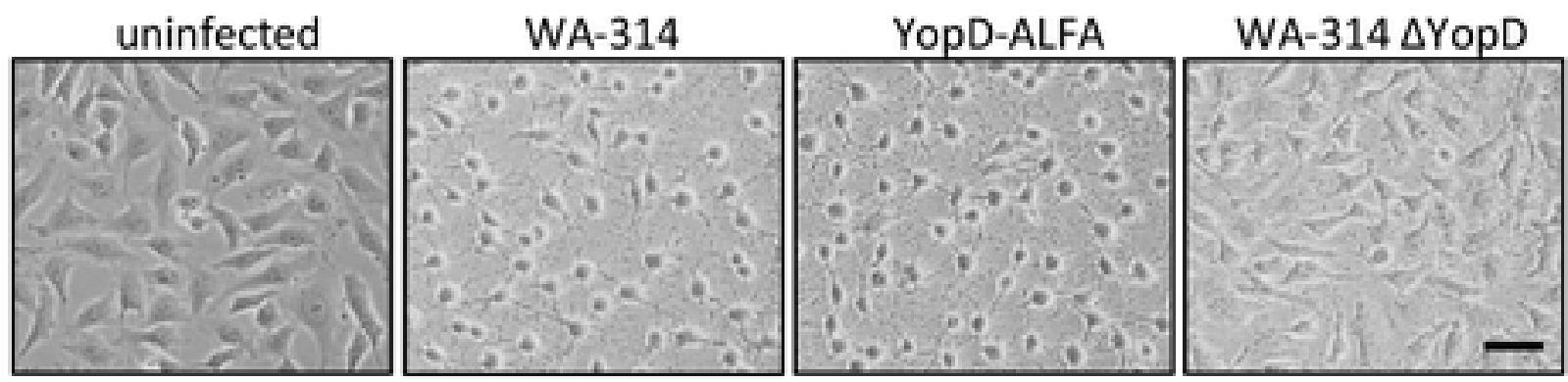




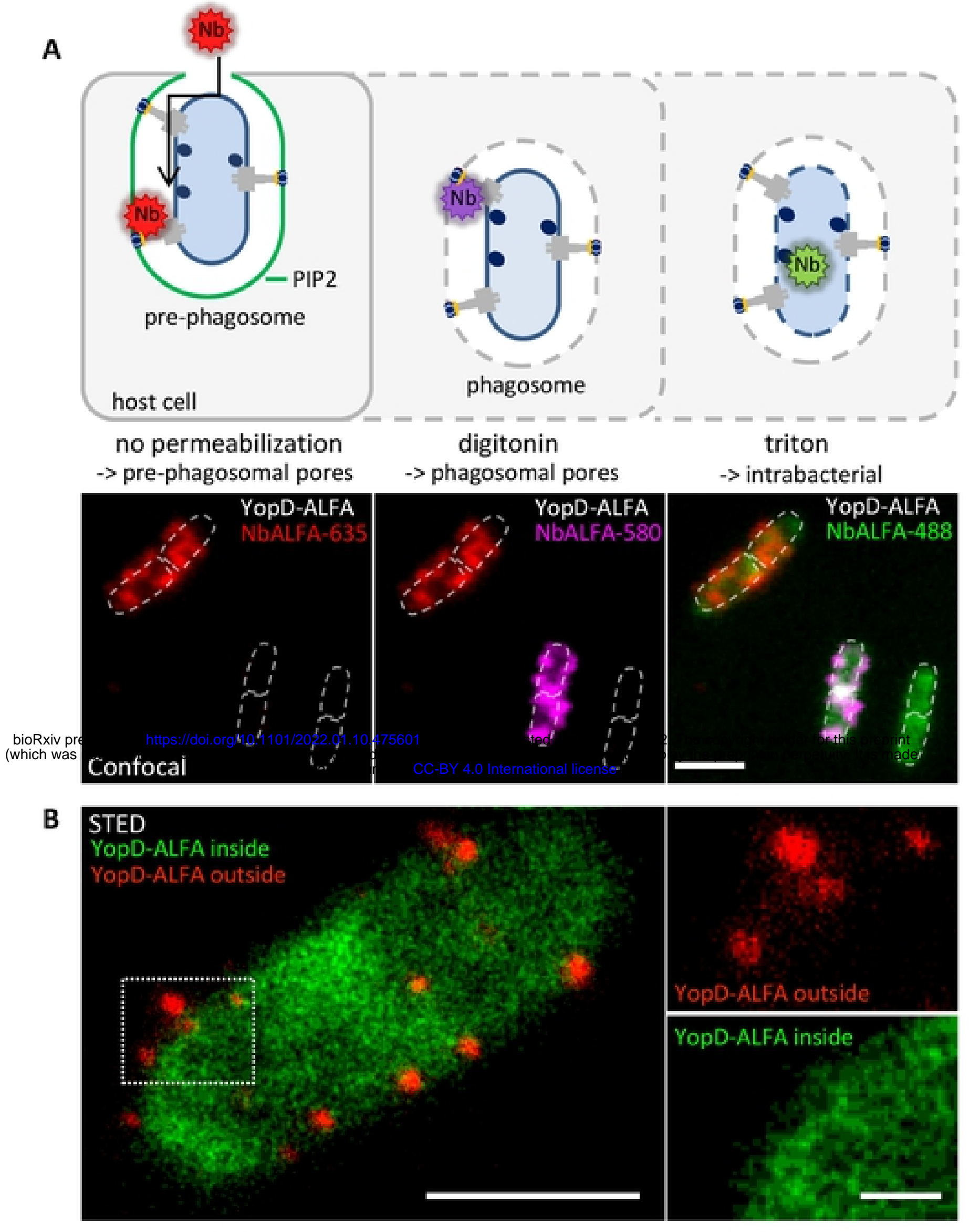



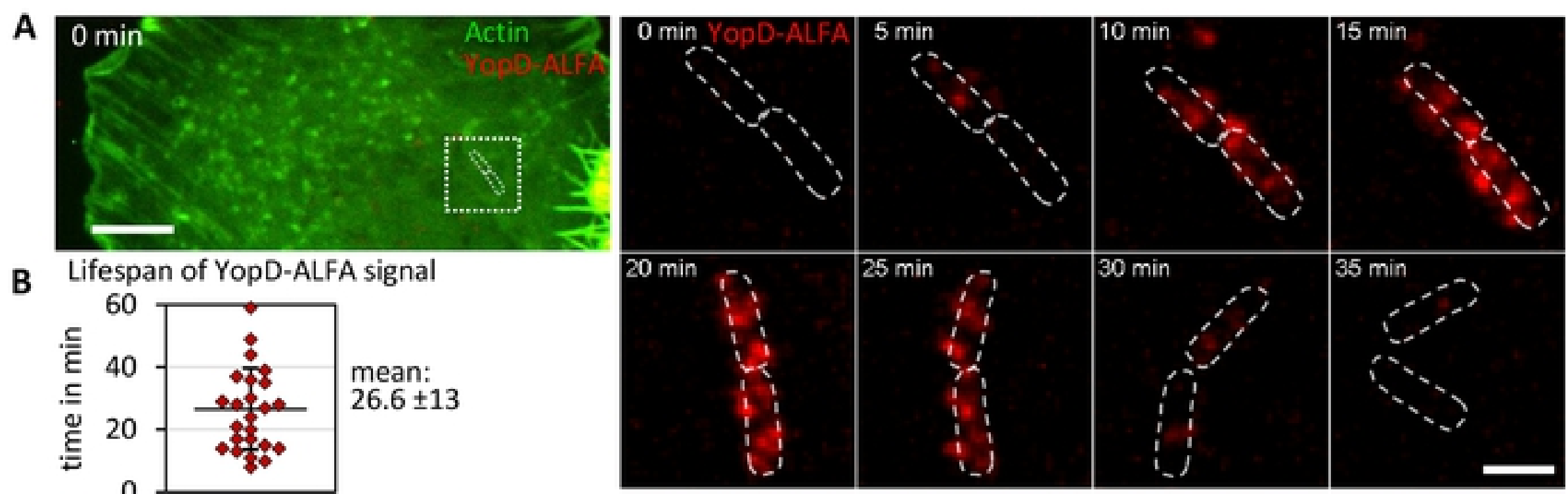

B Lifespan of YopD-ALFA signal
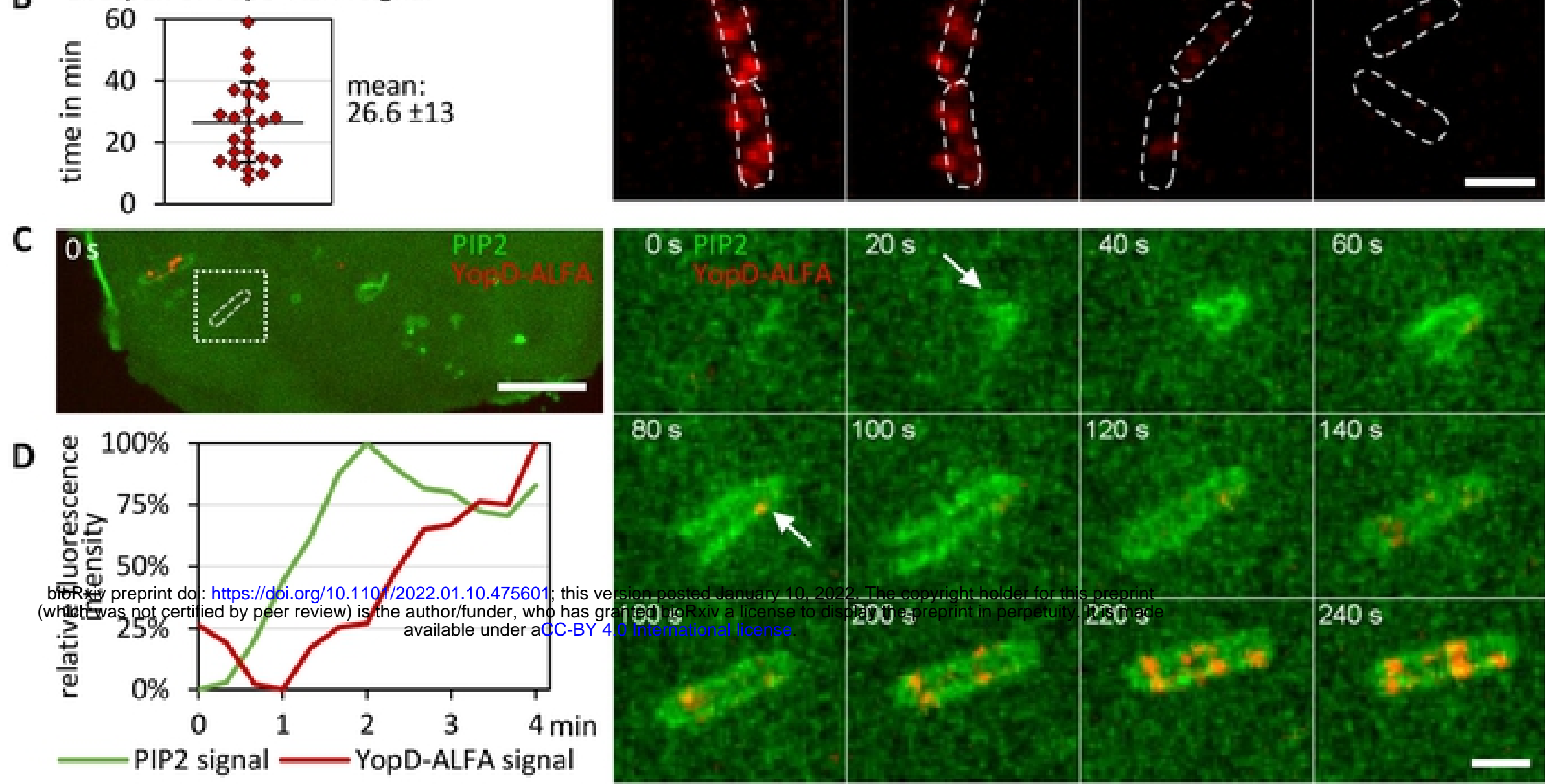

E PIP2 appearance in relation $\mathbf{F}$

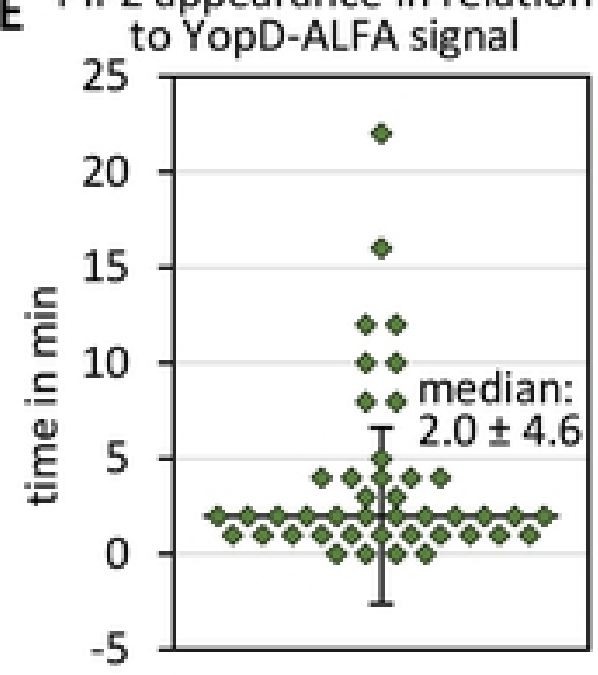

Primary human macrophages
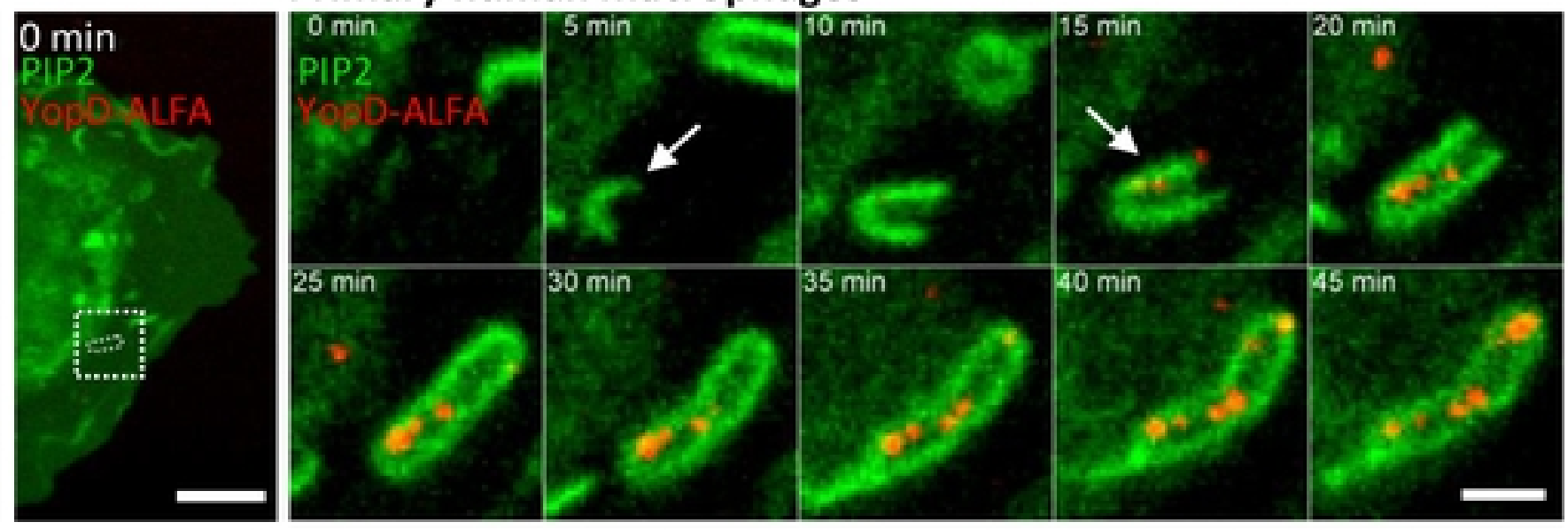
A

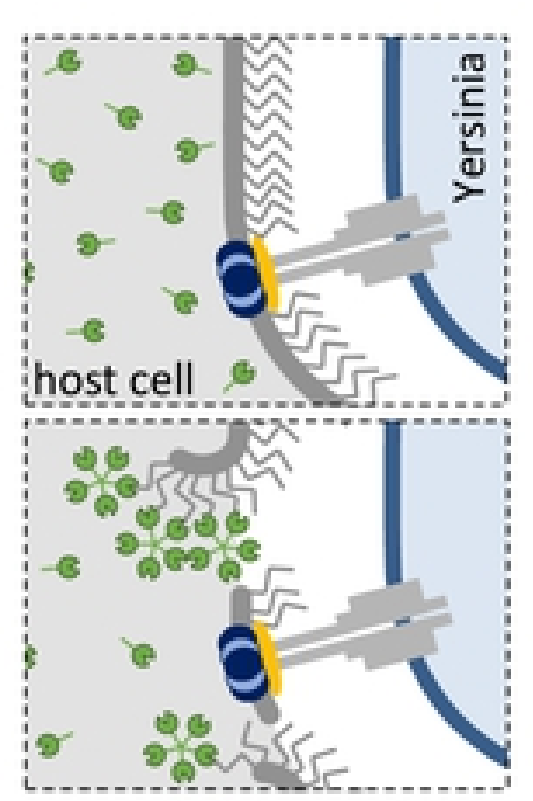

Sglycan eGFP-galectin-3
B galectin-3 positive bacteria $\%$ cell associated

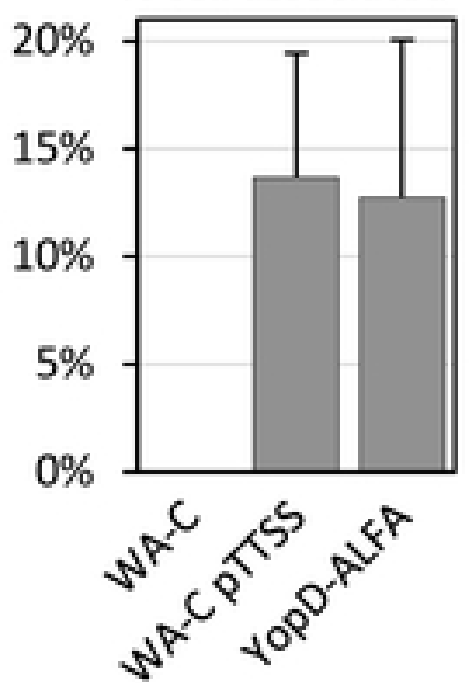

C

galectin-3 recruitment to phagosome

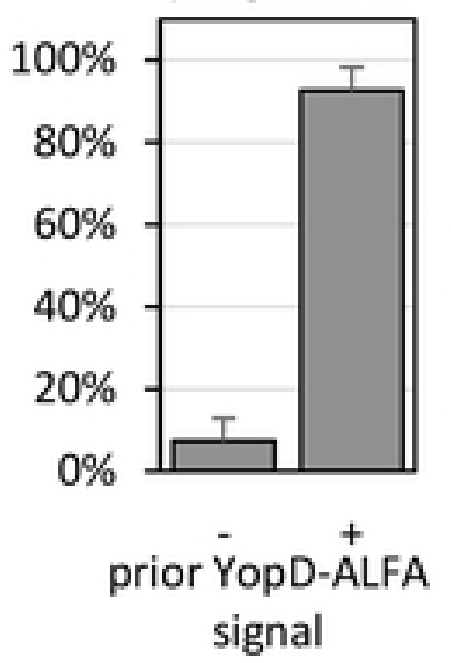

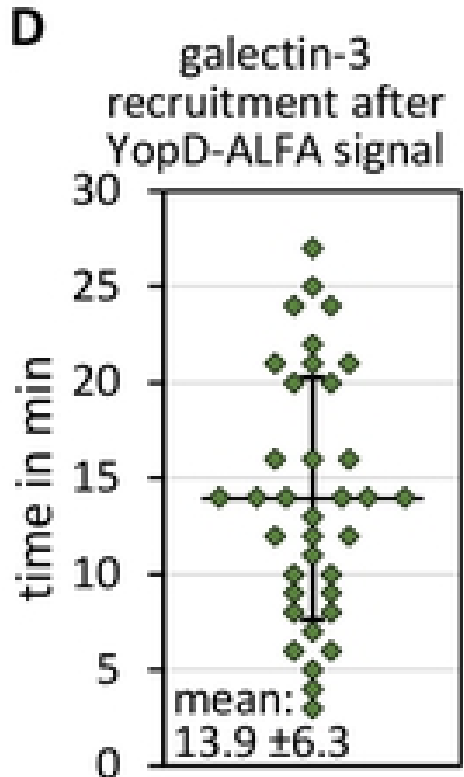

$13.9 \pm 6.3$
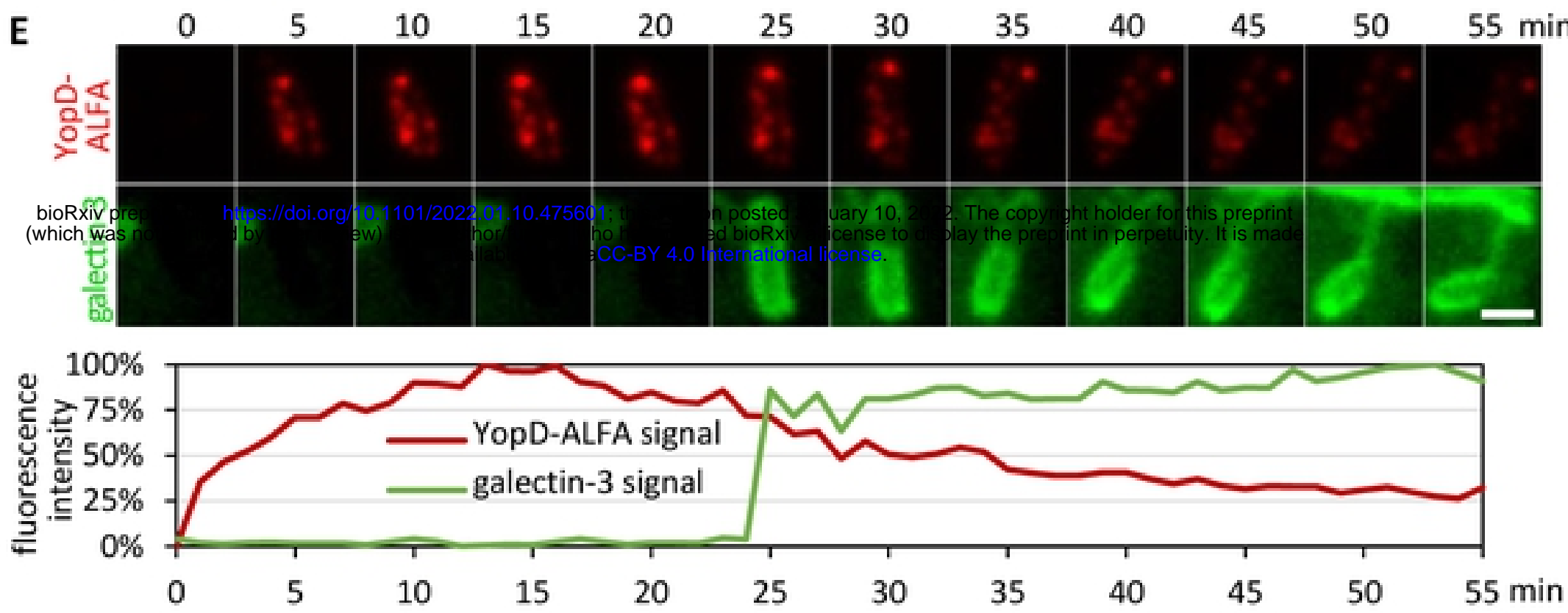

F YopD-ALFA signal reduction after galectin-3 recruitment

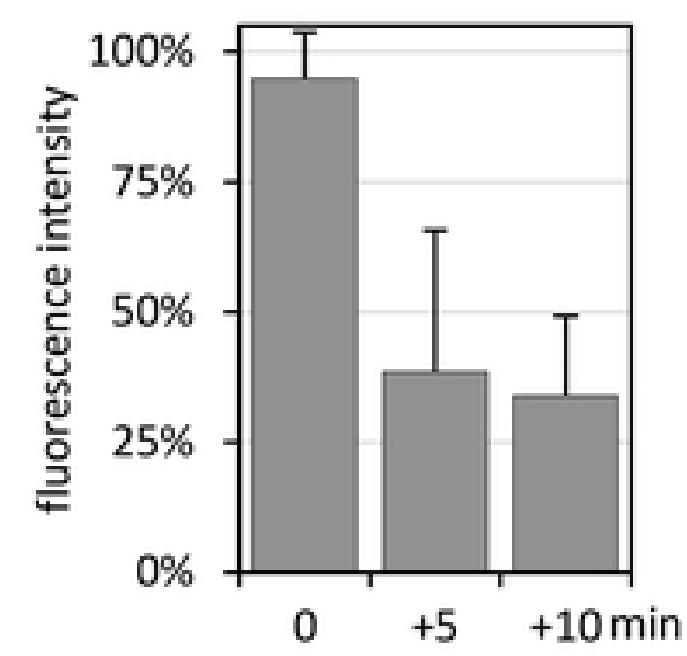

\section{G}

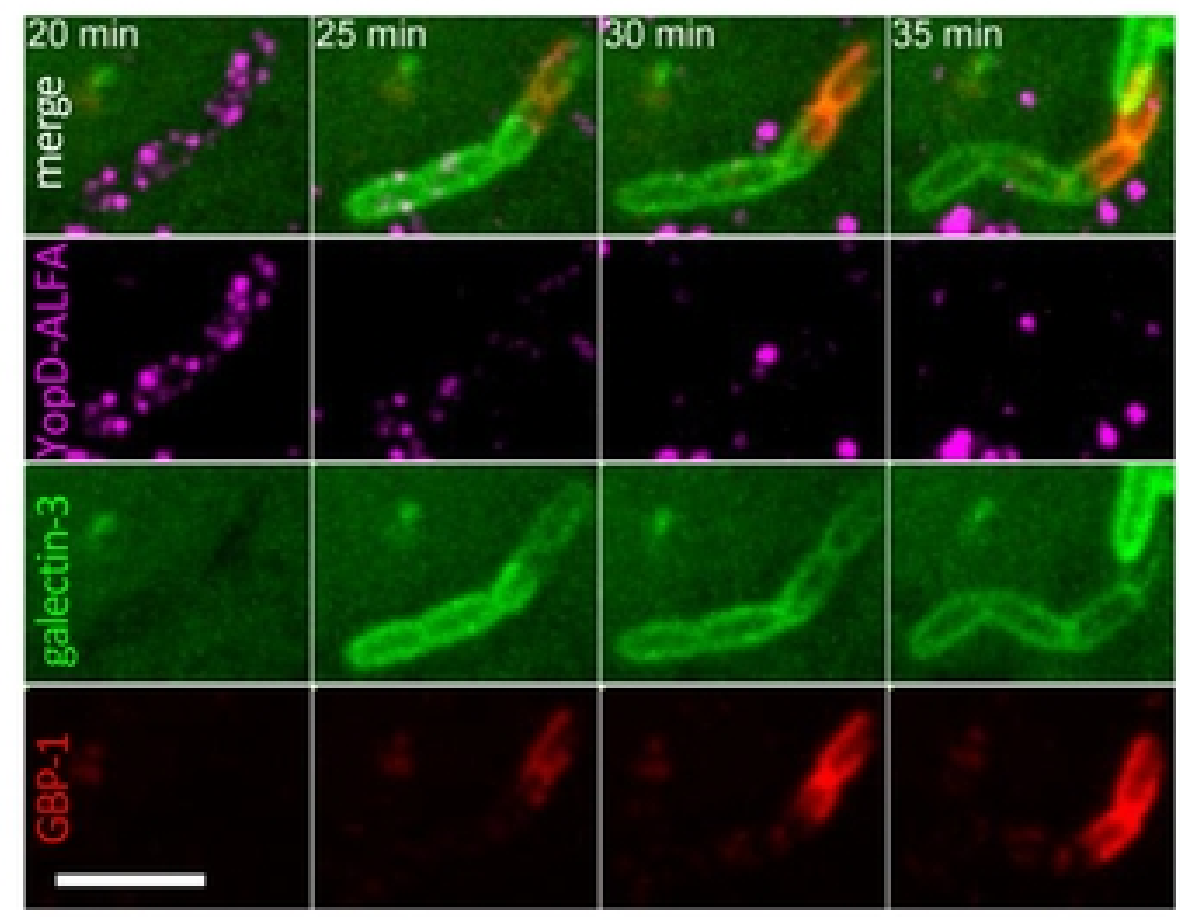

H

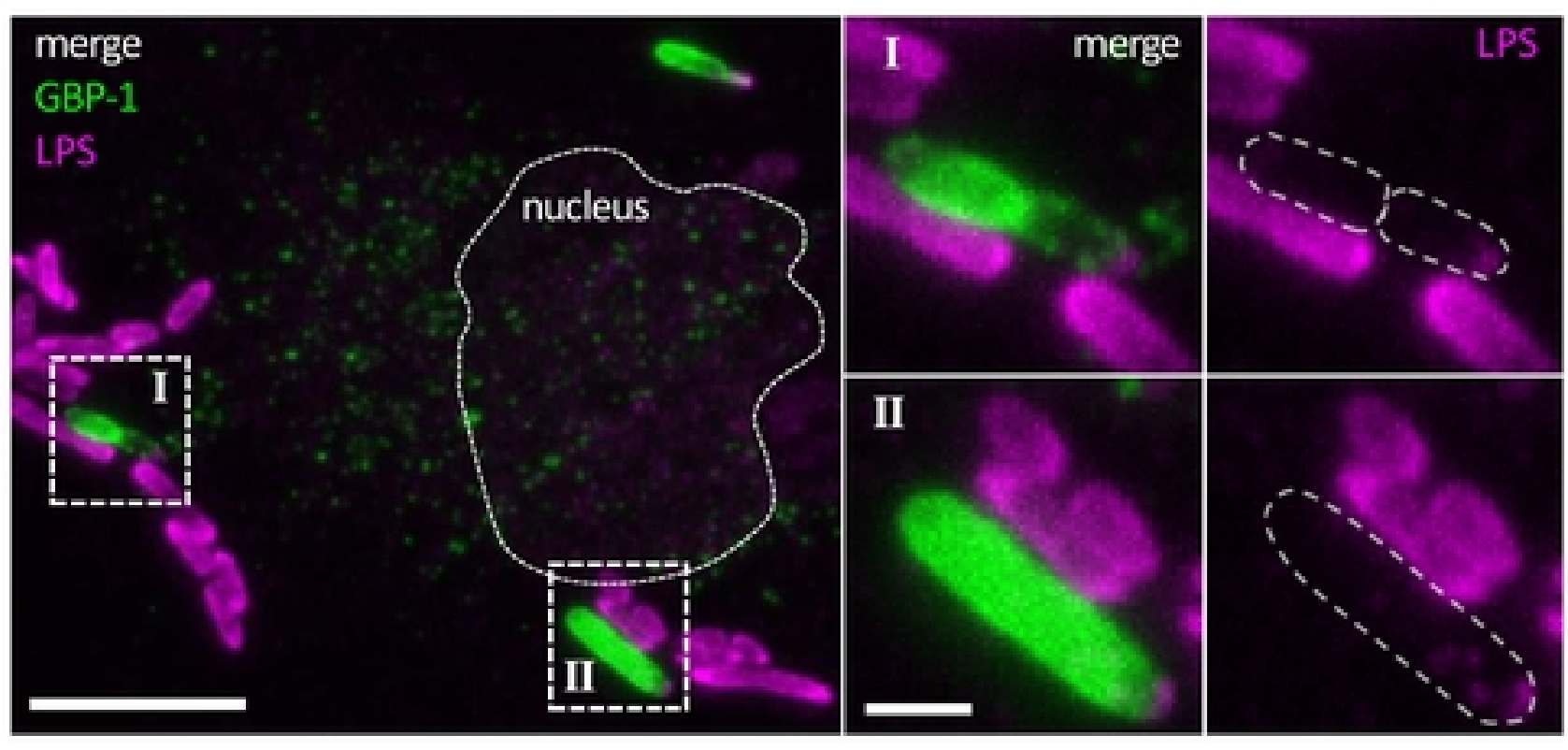

